\title{
Distances on Cosmological Scales with VLTI
}

\author{
Margarita Karovska, Martin Elvis, and Massimo Marengo \\ Harvard-Smithsonian Center for Astrophysics
}

\begin{abstract}
.
We present here a new method using interferometric measurements of quasars, that allows the determination of direct geometrical distances on cosmic scales. Quasar Broad Emission Line Regions sizes provide a 'meter rule' with which to measure the metric of the Universe. This method is less dependent of model assumptions, and even of variations in the fundamental constants (other than c). We discuss the spectral and spatial requirements on the VLTI observations needed to carry out these measurements.
\end{abstract}

\section{Introduction}

Determining the 3-D geometry of the Universe involves measurement of the large "cosmic" scale distances of high redshift sources such as distant supernovae and quasars. Cosmic distance scale determination methods include relative and absolute distance estimators. Absolute distance estimators include various applications of the: BaadeWesselink method (eg. Sasselov and Karovska 1994; Marengo et al., this workshop), and distances estimators using time delays (eg. SN1987A; Panagia et al. 2001). Relative distance estimating methods include period-luminosity relation for Cepheid star variability (used by the Hubble Key Project ; Freedman et al. 2001), and supernovae of type SN1a as 'standard candles'. Most strikingly, recent distance measurements using the brightness of supernovae of type SN1a at z 1.5 as 'standard candles' suggest that that the universe has a non-zero cosmological constant, $\Lambda$, so that the expansion of the Universe is currently accelerating (Perlmutter et al. 1999).

Relative distance estimators often involve assumptions and correlations, and have inevitable model dependencies. Absolute methods on the other hand have the advantage of having lesser dependence on physical models and provide an independent way to determine the distance scale.

We describe here an absolute method for estimating distances to quasars at different $z$ using a time delay measurements of linear sizes of quasars Broad Emission Line Regions (BELRs, Peterson 1997) and measurements of their angular sizes using large baseline interferometers such as the VLTI. This method could allow determining direct

(C) 2018 Kluwer Academic Publishers. Printed in the Netherlands. 
(a)

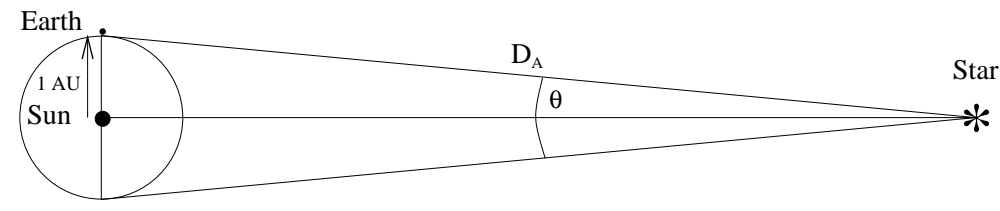

(b)

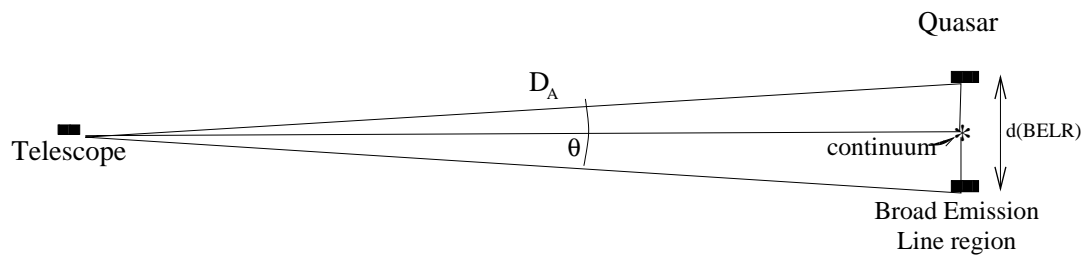

Figure 1. Measuring geometric distances to astronomical objects: (a) parallax to a star; (b) broad emission line region distance to a quasar.

geometrical distances to radio-quiet quasars and therefore measuring the cosmological constant, $\Lambda$, with minimal assumptions.

\section{Quasar Parallax}

We propose to use a new geometric method to determine distances to quasars that is equivalent to the parallax method. However, in this case instead of using the Earth's orbit as a known 'standard length' (Figure 1a), we use the size of the quasar BELR (Figure 1b) as measured using reverberation mapping and interferometry (Elvis and Karovska 2002).

The size of the BELR is known from light travel time measurements. BELRs emit a series of strong lines from permitted transitions, indicating Doppler velocities of $5000-10,000 \mathrm{~km} \mathrm{~s}^{-1}$ — defining characteristic of quasars. The Broad Emission Lines (BELs) respond to changes in the continuum source in the center of the quasar by changing their intensity with a time-lag of a few days or more (for higher luminosity and redshift). The time-lag is induced by the light travel time from the continuum source in the center of the quasar to the gas clouds in the BELRs that produce the broad emission lines (Peterson 1993, 2001). By studying the response of the BELs to continuum changes it is possible to map out the structure of the region emitting the BELs. This method is called 'reverberation mapping'.

Reverberation mapping exists for a few tens of active galaxies and quasars. The size of the broad emission line region depends on the line 
measured. Observations indicate that a single timescale dominate for each emission line (Nezter \& Peterson 1997). For low redshift quasars the size of the BELRs is $\sim 10$ light days. The timescale of BEL variations increases with redshift as $(1+z)^{2.5}$, since not only does the BELR size increase, but also the intrinsic variation timescale is dilated by $(1+z)$. Typical reverberation time for quasars at $\mathrm{z}=3$ requires observing programs spanning of 3-5 years.

The 'angular diameter distance' derived using the reverberation mapping and the interferometric measurements can be mapped as a function of redshift. This relation will describe the basic characteristic of the space-time metric of the Universe, and could eventually allow a derivation of $\Lambda$ (Elvis and Karovska 2002). This method of derivating $\Lambda$ is much less dependent on physical models and of changes in the fundamental constants (other than $c$, the speed of light) because it uses a 'standard length' approach rather than a 'standard candle'.

\section{Interferometry of Quasar BELRs}

Measurements of the angular sizes of BELRs are not easy even for the nearby quasars and AGNs. For example, at $\mathrm{z}<0.2$ the BELRs have measured lags that imply angular sizes $\sim 0.1$ mas (Fig 2.). This angular size is about 10 times smaller then the baselines of the existing ground based interferometers operating in optical and near-IR wavelengths (e.g. NPOI, IOTA). In addition, their sensitivity and the limiting magnitude are far bellow what is required for measuring the BELRs.

To measure accurately the sub-milliarcsecond scale BELR angular diameters therefore requires very long-baseline interferometers with large aperture telescopes, and with a capability of observing in selected quasar emission lines. In addition, the baseline of an interferometer that can resolve the BELR increases with $z$ for a fixed physical size, because the observed wavelength of any emission line increases as $(1+z)$.

Reverberation mapping suggests that shorter wavelength emission lines (in the UV or X-ray bands) may be more favorable for BELR size measurements. However, the space-based interferometers operating at sub-optical wavelength range are currently only in planning stages, and the ground-based interferometry is at present the only mean to carry out these measurements.

Several long-baseline ground-based optical and near-IR interferometers that have a potential of measuring the sizes of the BELRs in nearby quasars and AGNs are currently being built or commissioned. These interferometers, including the VLTI, are employing large aper- 


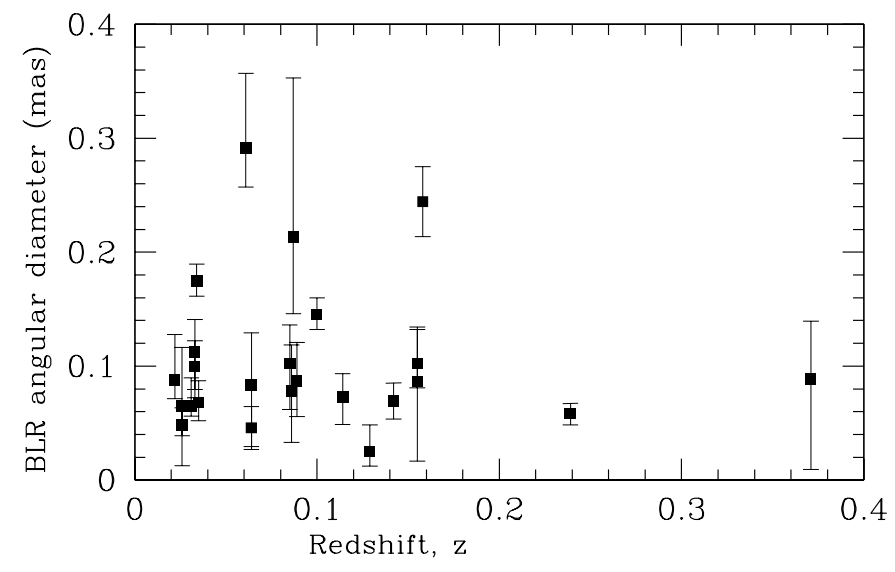

Figure 2. Angular diameters for the $\mathrm{H} \alpha$ and $\mathrm{H} \beta$ BELRs of nearby active galaxies, assuming $\mathrm{H}_{0}=65 \mathrm{~km} \mathrm{~s}^{-1} \mathrm{Mpc}^{-1}$ (Peterson et al. 1998, Kaspi et al. 2000). (The values of $\Omega$ and $\Lambda$ are unimportant at these low redshifts.

ture telescopes (e.g. VLTI $1.8 \mathrm{~m}$ to $8-\mathrm{m}$ ) on baselines of several hundred meters and have the sensitivity required to observe BELRs in selected spectral lines used in the reverberation measurements.

Since lag-time and angular size measurements must be carried out using the same emission lines, we are currently exploring optical and near-IR lines that may be suitable for reverberation mapping studies as well as for interferometry. Our initial studies indicate that in the optical $\mathrm{H} \beta, \mathrm{H} \alpha$ lines could be appropriate for these measurements. The next generation VLTI instruments operating in the optical would be able to resolve the nearby AGN BELRs and study their geometry. This is very important, because the geometry of quasar BELRs is not well known. Possibilities range from a simple orderly wind (Elvis 2000) to a maximally chaotic distribution of clouds (Baldwin et al. 1995).

In the near-IR the VLTI could measure the BELRs sizes of nearby (very low redshift) unobscured AGNs (eg. with AMBER; Petrov et al.

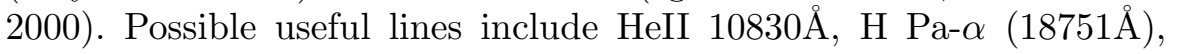


and $\mathrm{H} \mathrm{Pa}-\beta$ (12818 $\AA$ ). For the nearest AGNs (eg. Cen A) the expected BELRs sizes are significantly larger and therefore one could carry out imaging and study their geometry (Paresce 2001). For more distant AGNs and quasars one can then use this information to estimate the size of their BELRs. Our simulations show that using high-signal to noise visibility measurements (less then 1\%) and a model brightness distribution, one can significantly exceed the limiting resolution of the VLTI and estimate diameters much smaller then 1-2 mas. By assuming a model for the geometry of the BELRs the VLTI observations could therefore determine the distances to the nearby AGNs and quasars, and improve the $H_{0}$ accuracy to $\sim 5 \%$.

However, there are a number of issues regarding the feasibility of the interferometric measurements that need to be addressed. For example, there is an inherent problem in relating reverberation lag times as currently measured to the angular sizes that an interferometer would measure. Even perfectly sampled data will produce a broadened crosscorrelation function $(\mathrm{CCF})$, and it is not obvious which delay time, e.g. the CCF peak, or the CCF centroid, corresponds to the angular size from an image. The reverberation mapping measures a 'responsivity weighted' size of the light delay surface, while interferometry measures an 'emission weighted' size.

The amplitude of variation of the emission lines is much less than that of the continuum (eg. factor 2 changes in the UV continuum produce only $20 \%$ changes in the emission lines) By monitoring a number of quasars for continuum changes, one could identify the onset of a significant continuum increase in a particular quasar, which will then trigger 'target of opportunity' VLTI measurements. The VLTI could observe the target quasar in both low and high continuum states, with appropriate lags included, so that the difference map will be a measure only of the 'responsive fraction' of the line, i.e. that part which responds to continuum changes. Evidently, the best approach would be to continuously monitor quasars with the VLTI and derive the lag times and sizes from the same data.

\section{Conclusions}

Quasar BELRs provide a meter rule for determining the cosmic distance scale independent of changes in the fundamental constants (other than $c$, the speed of light). We describe a new absolute distance estimating method using reverberation time-lag measurements of the linear BELR sizes, combined with the VLTI interferometric angular size measurements, to determine the distances to quasars. VLTI can measure the 
sizes of BELRs and explore their geometry at low redshifts. This will provide input to BELR models and allow measurement of $H_{0}$. Longbaseline interferometers with a resolution of 0.01 mas are needed to measure the size of the BELR in $\mathrm{z}=2$ quasars, and therefore the $3-\mathrm{D}$ geometry of the Universe (and $\Lambda$ ) which appear plausible with near future extrapolations of current technology.

\section{Acknowledgements}

This work was supported in part by NASA contract NAS8-39073 (Chandra X-ray Center).

\section{References}

Baldwin J.A., Ferland G., Korista K. \& Verner D., 1995, ApJL, 455, L119

Elvis M., 2000, ApJ, 545, 63

Elvis M., 2002, Karovska M., ApJ Letters, in press.

Freedman W., et al., 2001, ApJ, 553, 47

Kaspi S., Smith P.S., Netzer H., Maoz D., Jannuzi B.T., \& Giveon U., 2000, ApJ, 533, 631 .

Netzer H. \& Peterson B.M., 1997, "Astronomical Time Series", eds. D. Maoz, A. Sternberg, and E.M. Leibowitz, 1997 (Dordrecht: Kluwer), p. 85.

Panagia N., Gilmozzi R., Macchetto F., Adorf H.-M., \& Kirshner R.P., 2001, ApJ, 380, L23

Perlmutter S., et al., 1999, ApJ, 517, 565

Peterson B.M., 1993, PASP, 105, 247

Peterson B.M., 1997, 'An Introduction to Active Galactic Nuclei' [Cambridge:CUP]

Peterson B.M., Wanders I., Betram R., Hunley J.F.; Pogge R.W., Wagner, R.M., 1998, ApJ, 501, 82

Peterson B.M., 2001, in 'The Starburst-AGN Connection 2001', [Singapore: World Scientific] astro-ph/0109495

Paresce, F., 2001, "Science with VLTI", (http://www.eso.org/projects/vlti/).

Petrov R. et al., 2000, Proc. SPIE Vol. 4006, 68, 'Interferometry in Optical Astronomy' eds: P.J. Lena and A. Quirrenbach. http://buz.obs-nice.fr/amber/),

Sasselov, D. \& Karovska, M., 1994, ApJ, 432, 367. 